\title{
Spatio-temporal linear stability of double-diffusive two-fluid channel flow
}

\author{
Kirti Chandra Sahu ${ }^{1, a)}$ and Rama Govindarajan² \\ ${ }^{1}$ Department of Chemical Engineering, Indian Institute of Technology Hyderabad, \\ Yeddumailaram 502 205, Andhra Pradesh, India \\ ${ }^{2}$ Engineering Mechanics Unit, Jawaharlal Nehru Centre for Advanced Scientific Research, \\ Jakkur, Bangalore 560 064, India
}

(Received 12 January 2012; accepted 19 April 2012; published online 15 May 2012)

\begin{abstract}
Absolute instabilities in shear flows can cause a catastrophic breakdown into a new unsteady state, or even into turbulence. We demonstrate that in a double-diffusive channel flow with a viscosity stratification across the channel, rapidly growing absolute instability may be obtained at Reynolds numbers of a few hundreds. The instability is much weaker in an equivalent single solute fluid with the same viscosity contrast, or even in one which is made up only of the more dangerous of the two diffusing species. This is a novel characteristic of double-diffusive systems driven by viscosity, rather than density variations. Convective instabilities too are stronger in the double-diffusive case. (일 2012 American Institute of Physics. [http://dx.doi.org/10.1063/1.4718775]
\end{abstract}

\section{INTRODUCTION}

The stability of core-annular flow through a channel of two miscible fluids is now a well-studied problem, see, e.g., Refs. 1-7. A schematic of this flow, in a situation where there is a thin mixed layer between the two fluids, is shown in Figure 1. When the two fluids are of the same density, and differ only in their viscosity, it is known ${ }^{1,5,6}$ that if the more viscous fluid is in the annular region, the laminar flow is greatly destabilized, whereas if the more viscous fluid occupies the core, a large stabilization typically results, unless the diffusivity is very small. The former case has also been shown to support regimes of absolute instability. ${ }^{8,9}$ Our present focus is on absolute instability of a two-solute flow, so we begin with a brief discussion on this.

In an absolutely unstable flow, ${ }^{10}$ disturbances grow locally as well as spread both upstream and downstream. Thus, eventually the entire flow regime becomes unstable and the system behaves as a self-sustained resonator, oscillating at an intrinsic frequency. ${ }^{11,12}$ Such a flow is also referred to as being globally unstable. ${ }^{10}$ In contrast, in a convectively unstable flow, disturbances amplify as they advect downstream, away from their initial location. In the three-layer miscible channel flow of Figure 1, Sahu et al. ${ }^{8}$ investigated convective and absolute instabilities with uniform layers of more viscous fluid coated on the channel walls with the less viscous fluid in the core of the channel. Identifying boundaries between convectively and absolutely unstable flows in the space of relevant parameters, they showed the gradients of viscosity perturbations in the wall-normal direction to be the main destabilizing influence. A similar flow of two fluids, in a pipe rather than a channel, was studied by Selvam et al. ${ }^{9}$ They found absolute instability at high ratios of annular to core viscosity. In this regime, their nonlinear simulations reveal self-excited intrinsic oscillations associated with the global mode.

The studies above were for two fluids, which may equivalently be thought of as being a pure solvent, and a solution containing a solute at a particular concentration. We refer to this below as a single component (SC) flow. When the viscosity stratification results from not one, but two solutes

\footnotetext{
a) ksahu@iith.ac.in.
} 

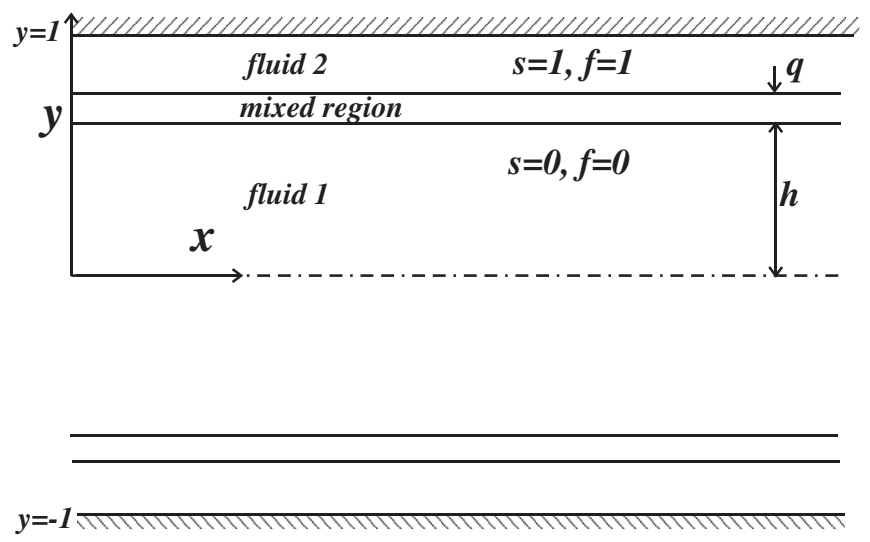

FIG. 1. Schematic of the three-layer base state flow. The fluids "1" and "2" occupy the channel core and the region adjacent to the channel walls, respectively. The two fluids are separated by a mixed layer of uniform thickness $q$, with fluid " 1 " located in the region $-h \leq y \leq h$.

of vastly different diffusivities, we have a double-diffusive (DD) situation. Surprising behaviour can result in this situation. Double-diffusive flows are often encountered in nature and in industry. In the food industry, milk at various concentrations, sugar syrup, and water with added thickeners are examples of fluids whose density does not vary much with concentration and temperature but the viscosity does. Both temperature and concentration gradients arise in the shear flows of these substances, and to make a homogeneous product, it can be beneficial if some degree of mixing is achieved in the pipe or channel flow portion of the process. Instabilities arising out of the DD nature of flows are well known in gravity-driven situations, see, e.g., Refs. 13-15. In contrast, the effect of viscosity contrasts in DD instabilities are less studied. Recently, Sahu and Govindarajan ${ }^{16}$ showed that in the two-fluid flow under discussion, two kinds of instability can result in a situation which is expected to be stabilizing, namely, when viscosity decreases towards the wall. The Reynolds numbers at which these instabilities occur are rather low, about 100. However, these instabilities were only convective, and searches over a wide range of parameters yielded no absolute instability.

In the context of obtaining good mixing of components at relatively low Reynolds numbers, one would wish to have absolute instability, which would be expected to lead to a catastrophic breakdown of the steady laminar flow. This is the focus of the present paper, where we study the double-diffusive instability of the case when the viscosity increases towards the wall. That this case is more unstable than the constant viscosity fluid flow through a channel is no surprise. We however do find a non-intuitive result, as follows. Most important, the DD flow is both convectively and absolutely unstable over a much wider range of parameters than a SC flow, and the growth rates, both convective and absolute, are significantly higher. In a SC flow, as the diffusivity of the solute in the solvent decreases, i.e., the Schmidt number increases, one expects, and finds, that the flow becomes increasingly unstable, with the instability setting in at very low Reynolds numbers. ${ }^{4}$ For $\mathrm{DD}$, the regime of instability increases even when the second solute is one which decreases the average Schmidt number.

\section{FORMULATION}

The spatio-temporal linear stability analysis of pressure-driven three-layer flow of two miscible, Newtonian and incompressible fluids of equal density and different viscosities in a horizontal, planar channel is studied. The description of the flow, the base state, and the stability equations are available in Ref. 16 but are included here for completeness. Fluid containing scalars $S$ and $F$ of concentration $S_{1}$ and $F_{1}$, respectively, and of net viscosity $\mu_{1}$ (fluid 1 ) occupies the core of the channel. The outer fluid (fluid 2) consists of scalars $S$ and $F$ in quantity $S_{2}$ and $F_{2}$ and is of viscosity $\mu_{2}$. In between there is a mixed layer of thickness $q$. Here, $F$ and $S$ designate the faster and slower diffusing scalars. 
$\mathcal{D}_{f}$ and $\mathcal{D}_{s}$ are the diffusion rates of the faster and slower diffusing scalars, respectively, such that $\delta=\mathcal{D}_{f} / \mathcal{D}_{s}$; and by definition $\delta \geq 1$.

The Cartesian coordinate system is used to formulate the problem, where $x$ and $y$ denote the coordinates in the flow and the wall-normal directions, respectively. The channel walls are rigid and impermeable, and are located at $y= \pm H$ as shown in Fig. 1. The viscosity, $\mu$, is modeled as an exponential function of both scalars

$$
\mu=\mu_{1} \exp \left[R_{s}\left(\frac{S-S_{1}}{S_{2}-S_{1}}\right)+R_{f}\left(\frac{F-F_{1}}{F_{2}-F_{1}}\right)\right],
$$

where $R_{s}\left(\equiv\left(S_{2}-S_{1}\right) \partial(\ln \mu) / \partial S\right)$ and $R_{f}\left(\equiv\left(F_{2}-F_{1}\right) \partial(\ln \mu) / \partial F\right)$ are the log-mobility ratios of the scalars $S$ and $F$, respectively. The following scaling is employed to render the governing equations dimensionless:

$$
\begin{array}{r}
(x, y)=H(\widetilde{x}, \widetilde{y}),(q, h)=H(\widetilde{q}, \widetilde{h}), t=\frac{H^{2}}{Q} \widetilde{t},(u, v)=\frac{Q}{H}(\widetilde{u}, \widetilde{v}), p=\frac{\rho Q^{2}}{H^{2}} \widetilde{p}, \\
\mu=\widetilde{\mu} \mu_{1}, \widetilde{s}=\frac{S-S_{1}}{S_{2}-S_{1}}, \widetilde{f}=\frac{F-F_{1}}{F_{2}-F_{1}},
\end{array}
$$

where the tildes designate dimensionless quantities; $Q$ denotes the total volume flow rate per unit distance in the spanwise direction; $u$ and $v$ are the velocity components in the $x$ and $y$ directions, respectively; $\rho$ is the constant density; $t$ is time and $p$ denotes pressure. Now dropping tildes for convenience, the dimensionless governing equations are given by

$$
\nabla \cdot \mathbf{u}=0
$$

$$
\begin{gathered}
{\left[\frac{\partial \mathbf{u}}{\partial t}+\mathbf{u} \cdot \nabla \mathbf{u}\right]=-\nabla p+\frac{1}{\operatorname{Re}} \nabla \cdot\left[\mu\left(\nabla \mathbf{u}+\nabla \mathbf{u}^{T}\right)\right],} \\
\frac{\partial s}{\partial t}+\mathbf{u} \cdot \nabla s=\frac{1}{\mathrm{Pe}} \nabla^{2} s \\
\frac{\partial f}{\partial t}+\mathbf{u} \cdot \nabla f=\frac{\delta}{\mathrm{Pe}} \nabla^{2} f
\end{gathered}
$$

where $\mathbf{u}$ is the velocity vector, $\operatorname{Re}\left(\equiv \rho Q / \mu_{1}\right)$ and $\operatorname{Pe}\left(\equiv Q / \mathcal{D}_{s}\right)$ are the Reynolds number and Péclet number, respectively. The Péclet number ( $\equiv \mathrm{ReSc}$ ) is defined based on the slower diffusing species, where $\mathrm{Sc}$ is its Schmidt number. The effective Schmidt number of the faster diffusing fluid is $\mathrm{Sc} / \delta$.

\section{A. Base state}

The base state, about which linear stability characteristics will be analyzed, corresponds to a steady, parallel, fully developed flow, i.e., $V=0, U$ is a function of $y$ alone and $P$ is linear in $x$. The upper-case letters for the velocity components and pressure, and the subscript 0 for $s, f$ and viscosity are used to designate the base state. The locally parallel assumption for the base state is a very good approximation, since the mixed layer is diffusing very slowly, with a divergence angle scaling as $\mathrm{Pe}^{-1}$. In order to achieve this in an experiment, one would need a long channel, and a careful inlet design. For very large Sc, momentum would diffuse faster than concentration and one would achieve a base state profile which is nearly parallel over a long length of the channel. In this study, we prescribe identical mean concentration profiles for both solutes. This however may not be easy to achieve in an experiment, since the faster diffusing fluid will have a correspondingly thicker mixed layer. Our prescription is consistent with our primary objective, which is to bring out only the effect of different diffusivities on the disturbance growth. We have made computations with concentration profiles of different thicknesses as well, but do not discuss those results here. The two constant property fluids are thus separated by a mixed layer of uniform thickness $q$, with fluid " 1 " located in the region $-h \leq y \leq h$, and fluid "2" in the region $h+q<|y|<1$. 
A symmetry boundary condition is prescribed across the centerline. To obtained continuous profiles up to the second derivative, we assume that $s_{0}$ and $f_{0}$ vary as fifth order polynomials in the mixed layer. We confirm that other sufficiently smooth profiles give practically indistinguishable results from those presented here. The base state velocity profile, $U(y)$, is obtained by solving the steady, fully developed version of Eq. (4), subject to no-slip and no-flux conditions at the wall and the centerline of the channel, respectively. The nondimensional pressure gradient $d P / d x$ is obtained by using $\int_{0}^{1} U d y=1$. The functional dependence of dimensionless viscosity is given by

$$
\mu_{0}=e^{\left(R_{s} s_{0}+R_{f} f_{0}\right)} .
$$

The component having negative log-mobility ratio makes the viscosity decrease towards the wall, and is hence stabilizing, whereas the component with positive log-mobility ratio contributes a positive viscosity stratification (increases the viscosity towards the wall), which is destabilizing. A species with a null value of the log-mobility ratio does not contribute to the viscosity stratification.

\section{B. Linear stability analysis}

We examine the spatio-temporal linear stability of the base flow using a normal mode analysis. Our approach uses a Briggs-type method ${ }^{17}$ to obtain absolute growth rates. We decompose the flow variables and the concentration of the species into their steady base state parts and two-dimensional, linear perturbations, designated by a hat ${ }^{18}$

$$
(u, v, p, s, f)(x, y, t)=\left(U(y), 0, P, s_{0}(y), f_{0}(y)\right)+(\hat{u}, \hat{v}, \hat{p}, \hat{s}, \hat{f})(y) e^{\mathbf{i}(\alpha x-\omega t)} .
$$

Here, $\mathbf{i} \equiv \sqrt{-1}$, and $\alpha$ and $\omega$ are the wavenumber and frequency of the disturbance, respectively, both of which can be complex. The imaginary part, denoted by the subscript $i$, of $\omega$ gives the temporal growth or decay rate of the perturbation. The perturbation viscosity may be written as $\hat{\mu}=\frac{\partial \mu_{0}}{\partial s_{0}} \hat{s}+\frac{\partial \mu_{0}}{\partial f_{0}} \hat{f}$. In the usual simplification afforded by two-dimensional incompressible flow, the components of velocity are replaced by a single streamfunction, i.e., $(\hat{u}, \hat{v})=\left(\psi^{\prime},-\mathbf{i} \alpha \psi\right)$, where the prime denotes differentiation with respect to $y$. Substitution of Eq. (8) into Eqs. (3)-(6), linearization, and elimination of the pressure terms yields the linear stability equations

$$
\begin{aligned}
\mathbf{i} \alpha \operatorname{Re}\left[\left(\psi^{\prime \prime}-\alpha^{2} \psi\right)(U-c)-U^{\prime \prime} \psi\right]= & \mu_{0}\left(\psi^{i v}-2 \alpha^{2} \psi^{\prime \prime}+\alpha^{4} \psi\right) \\
& +2 \mu_{0}^{\prime}\left(\psi^{\prime \prime \prime}-\alpha^{2} \psi^{\prime}\right)+\mu_{0}^{\prime \prime}\left(\psi^{\prime \prime}+\alpha^{2} \psi\right) \\
& +U^{\prime}\left(\mu^{\prime \prime}+\alpha^{2} \mu\right)+2 U^{\prime \prime} \mu^{\prime}+U^{\prime \prime \prime} \mu, \\
\mathbf{i} \alpha \operatorname{Pe}\left[(U-c) s-\psi s_{0}^{\prime}\right] & =\left(s^{\prime \prime}-\alpha^{2} s\right), \\
\mathbf{i} \alpha \operatorname{Pe}\left[(U-c) f-\psi f_{0}^{\prime}\right] & =\delta\left(f^{\prime \prime}-\alpha^{2} f\right),
\end{aligned}
$$

where $c(\equiv \omega / \alpha)$ is the phase speed of the disturbance. Note that the hat notation is suppressed in the above equations. The boundary conditions are

$$
\begin{aligned}
& \psi=\psi^{\prime}=s=f=0 \text { at } y=1, \\
& \psi^{\prime}=\psi^{\prime \prime \prime}=s^{\prime}=f^{\prime}=0 \text { at } y=0 .
\end{aligned}
$$

The above boundary conditions are for symmetric mode. We performed computations for the antisymmetric mode as well, and found the symmetric mode to be always dominant in this range of parameters.

The eigenvalue problem described by Eqs. (9)-(11) along with the boundary conditions (12) and (13) is solved using Chebyshev spectral collocation ${ }^{19}$ on a stretched grid, as described in Ref. 16. In order to determine whether the flow is stable or unstable and, in the latter case, whether absolutely or convectively unstable, we follow the approach used previously to analyze the stability of mixing layers, jets and wakes, and in plasma flows. ${ }^{11,17,20-23}$ As mentioned earlier, 
this approach has also been applied to analyze convective-absolute instabilities in single-solute miscible $e^{8,9}$ two-layer channel flow. The procedure is briefly outlined below.

The linearized differential operator represents a dispersion relation in complex $(\omega, \alpha)$ space. The corresponding Green's function, $G(x, y, t)$, gives the response of the linearized system to an impulse perturbation. For a chosen mode, the long-time behaviour of $G$ along different "rays", along which $x / t$ is constant, is then analyzed. It may be noted that this quantity corresponds to the group velocity of the mode, i.e.,

$$
\frac{x}{t}=\frac{\partial \omega}{\partial \alpha}(\alpha)
$$

We now distinguish between the maximum temporal growth rate, $\omega_{i, \max }$, corresponding to a real wavenumber $\alpha$, and the imaginary part of the "absolute frequency," $\omega_{0}=\omega\left(\alpha_{0}\right)$, corresponding to the "absolute wavenumber" $\alpha_{0}$, at which the saddle point condition

$$
\frac{\partial \omega}{\partial \alpha}\left(\alpha_{0}\right)=0
$$

is satisfied. We have absolute instability if $\omega_{0 i}>0$, that is, if the absolute growth rate is positive. From Eqs. (14) and (15), it is clear that we then have an impulse response growing locally, and usually spreading both upstream and downstream from its source. On the other hand, if $\omega_{0, i}<0$ but $\omega_{i, \max }>0$, the impulse disturbances grow as they move downstream from their source, giving rise only to a convectively unstable flow.

\section{RESULTS AND DISCUSSION}

We begin by setting the wavenumber to a real value, $\alpha_{r}$, and evaluating the convective instability of SC and DD systems, both of which are more viscous close to the wall. Shown in Figure 2(a) is a typical result of the maximum temporal growth rate. In the DD case, the ratio of diffusivities $\delta$ has been chosen to be 10 and the Schmidt number to be 50, so the faster diffusing component $F$ diffuses at a rate 5 times slower than momentum, and the other component $S$ diffuses 10 times slower than $F$. The SC system has the same average diffusivity as the DD system $\mathcal{D}_{\mathrm{SC}}=\left(\mathcal{D}_{s}+\mathcal{D}_{f}\right) / 2$; so the equivalent $\mathrm{Schmidt}$ number of the $\mathrm{SC}$ flow is given by $2 \mathrm{Sc} /(1+\delta)$. All other parameters are held the same, but it is clear that, for this set of parameters, the DD is unstable with a significant growth rate, while the SC is not. That the DD is in general more easily destabilizable than the SC is reinforced by Figure 2(b) which shows the neutral boundaries for the two cases in the $\operatorname{Re}-\alpha_{r}$ plane. The DD flow displays instability at a far lower Reynolds number than the equivalent SC system, and is also unstable over a wider range of wavenumbers. The mechanism which is usually offered in
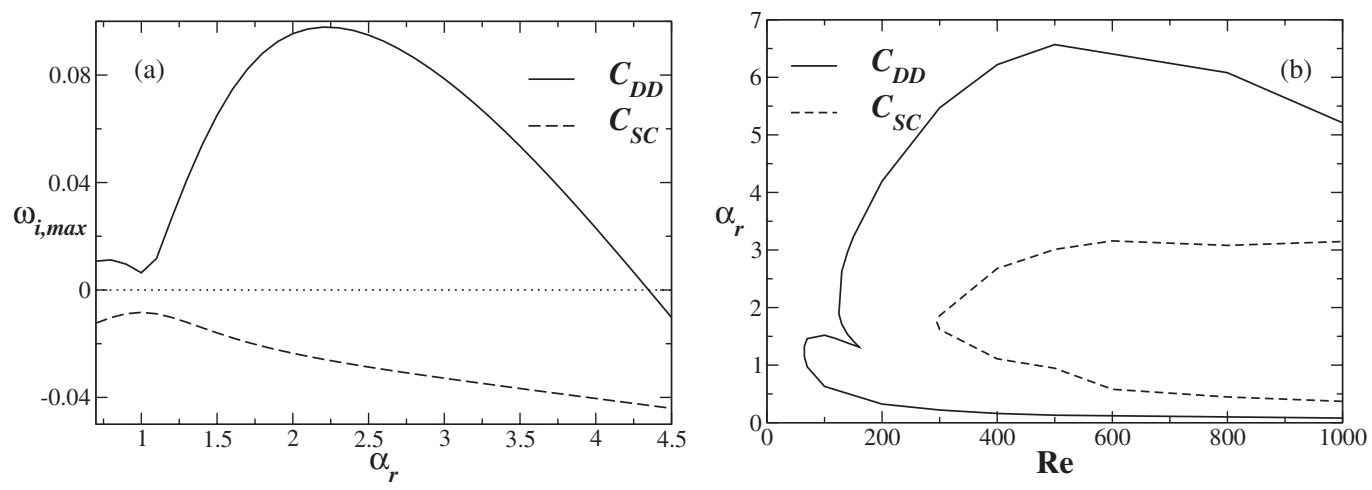

FIG. 2. Comparison of DD with SC convective instability for $h=0.6$ and $q=0.1$ : (a) dispersion curve ( $\omega_{i, \max }$ versus $\alpha_{r}$ ) for $\mathrm{Re}=200$, (b) neutral stability curve. Curves $C_{\mathrm{DD}}$, with $\mathrm{Sc}=50, R_{s}=5, R_{f}=-0.5$, and $\delta=10$ are for a DD system. The SC cases, denoted by $C_{\mathrm{SC}}$, are obtained by setting one of the stratification rates, $R_{f}$ in this case, to zero. To make the two systems equivalent, for the $\mathrm{SC}$ case we set $\mathrm{Sc}=9.091$ and $R_{S}=4.5$. To obtain the maximum convective temporal growth rate, we have set the imaginary part of the disturbance wavenumber, $\alpha_{i}=0$. 
the analogous gravity-driven DD instability (the fingering mode), is that perturbations in the faster diffusing (stabilizing in this case) species will be erased away sooner, leaving the slower diffusing species to determine long time behaviour.

The reverse case, where the slower diffusing species is stabilizing and the faster diffusing one destabilizes, is also known to give instability in the gravity-driven case (the oscillatory mode), but this instability is usually weaker than the fingering mode. We now examine this reverse case in the flow, with $R_{s}<R_{f}$, for convective instability. Figure 2 shows that the DD mode is now less unstable than the SC, which is broadly consistent with the arguments made in the gravity-driven case. We shall see, however, in the absolute instability results below, that the expectation that the slower diffusing species will dominate is not sufficient to explain the large increase in absolute instability.

To begin our discussion on absolute instability, we demonstrate, in Figures 4 and 5, a typical situation where the DD flow is absolutely unstable, whereas the equivalent SC flow is not. In fact, the absolute growth rate is significant in the DD case, while the SC mode with zero group velocity is damped out rapidly. In the DD flow shown, the slower diffusing component is destabilizing, while the faster diffusing one is stabilizing.

To test out the argument that the DD flow with $R_{s}>R_{f}$ would show the behaviour dictated by the slower diffusing species, we make another comparison with a SC fluid, but this time with one which has all the properties of the slower diffusing species. The result is shown in Figure 6. If one had to guess a winner for the more unstable flow, this may be termed an unfair comparison, since the average viscosity stratification of the SC fluid, as well as its average diffusion rate are now far higher than the DD fluid. Both these are loaded in favour of the SC fluid being more unstable. This case does show absolute instability, but its growth rate is six times lower than the DD case. In other words, the DD flow, which is expected from heuristic arguments to be the more stable, shows a much higher absolute instability, and is thus likely to degenerate more rapidly into a turbulent state.

The reverse case, where the faster diffusing species is destabilizing, and the slower one is stabilizing, did not display any absolute instability in the parameter range of our search. A typical example is shown in Figure 7. The flow was however seen earlier, in Figure 3 to be convectively unstable with a large growth rate of $\omega_{i, \max }=0.0842$.

By repeating the exercise of finding the absolute frequency for a wide range of viscosity ratios, we may summarise the effect of two different rates of diffusion as opposed to an equivalent single one. We present results for the case where the slower diffusing species is destabilizing, and the faster one stabilizing, since only this case shows absolute instability. The absolute growth rates for two different thicknesses of mixed layer are shown in Figure 8. To get an SC fluid we set $\delta=1$, i.e., equalise the rates of diffusivity of $F$ and $S$. In both cases, the DD system is clearly absolutely

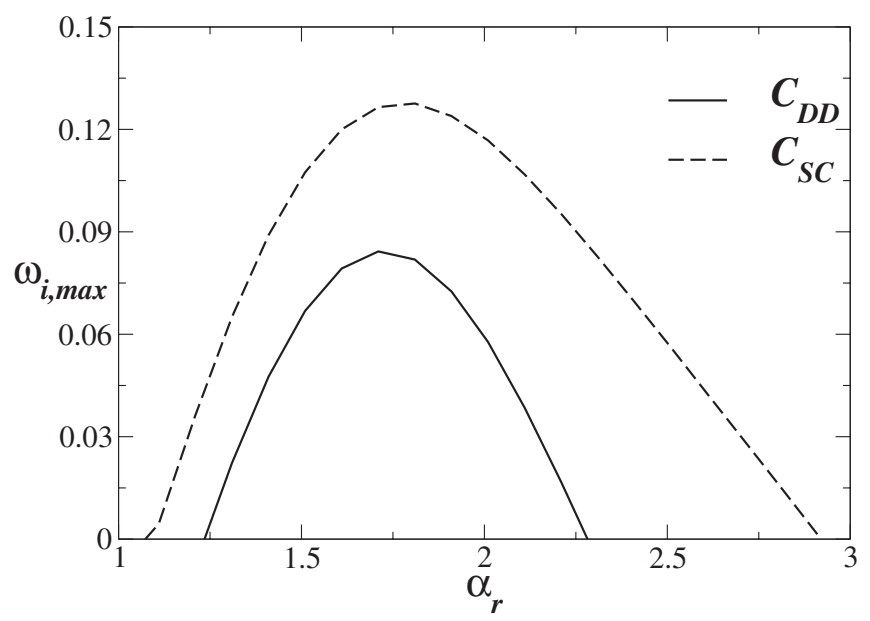

FIG. 3. Comparison of DD with SC stability for the case where the slower diffusing species is stabilizing, with $\operatorname{Re}=200$, $h=0.6$, and $q=0.1$. Curve $C_{\mathrm{DD}}$, with $\mathrm{Sc}=50, R_{s}=-0.5, R_{f}=4$, and $\delta=10$ is for a DD system, while $C_{\mathrm{SC}}$ with $\mathrm{Sc}=9.091, R_{s}=3.5$, and $R_{f}=0$ is for an SC fluid. Here, $\alpha_{i}=0$. 

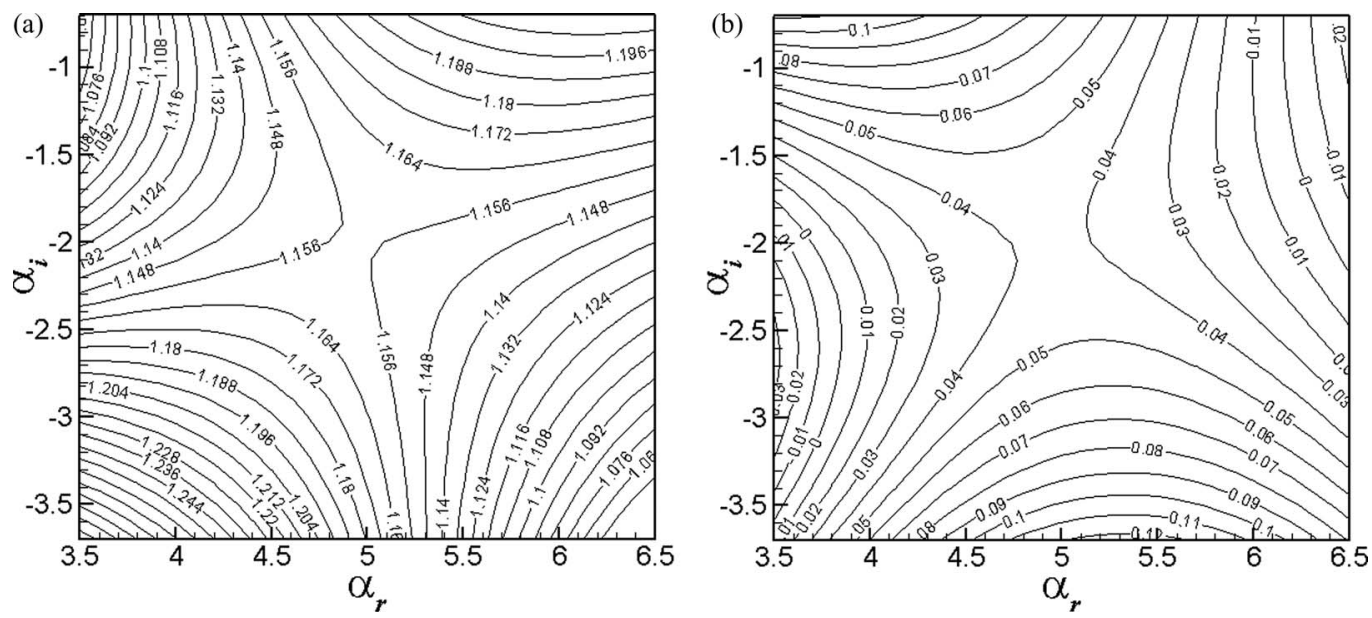

FIG. 4. Isocontours of (a) $\omega_{r}$ and (b) $\omega_{i}$ in the complex wavenumber plane for a DD system at $\mathrm{Re}=200$. The rest of the parameters are $\mathrm{Sc}=50, \delta=10, h=0.6, q=0.1, R_{s}=4$, and $R_{f}=-0.5$. The absolute frequency $\omega_{0}$, i.e., $\omega$ at the saddle point, is $1.156+0.041 \mathbf{i}$, showing absolute instability.

unstable over a wider range of viscosity ratio. Also the absolute growth rate is much higher. The difference between SC and DD is particularly stark in the case of a thicker stratified layer.

Finally, in Figure 9, we show the regimes of absolute and convective instability in the $R_{s}-R e$ plane for the DD flow. In the region above $R_{s}+R_{f}=0$, we have an "unstable" stratification of viscosity, i.e., the more viscous fluid occupies the annular region. Such a flow is convectively unstable above a Reynolds number of about 15 . In fact, the answer is not very sensitive to the strength of viscosity stratification. However, it may be noticed that the case of $R_{f}=-1$, which affords a net viscosity stratification which is lower than $R_{f}=-0.5$, is already convectively unstable for slightly lower values of $R_{s}$, indicating that the contrast in the two contributions enhances instability, and can be more important than the total stratification. At negative values of $R_{s}$, at the right hand lower corner of the figures, we have another region of convective instability. This is the DD instability in the "stably" stratified regime already discussed in Ref. 16. A wide region of absolute instability is
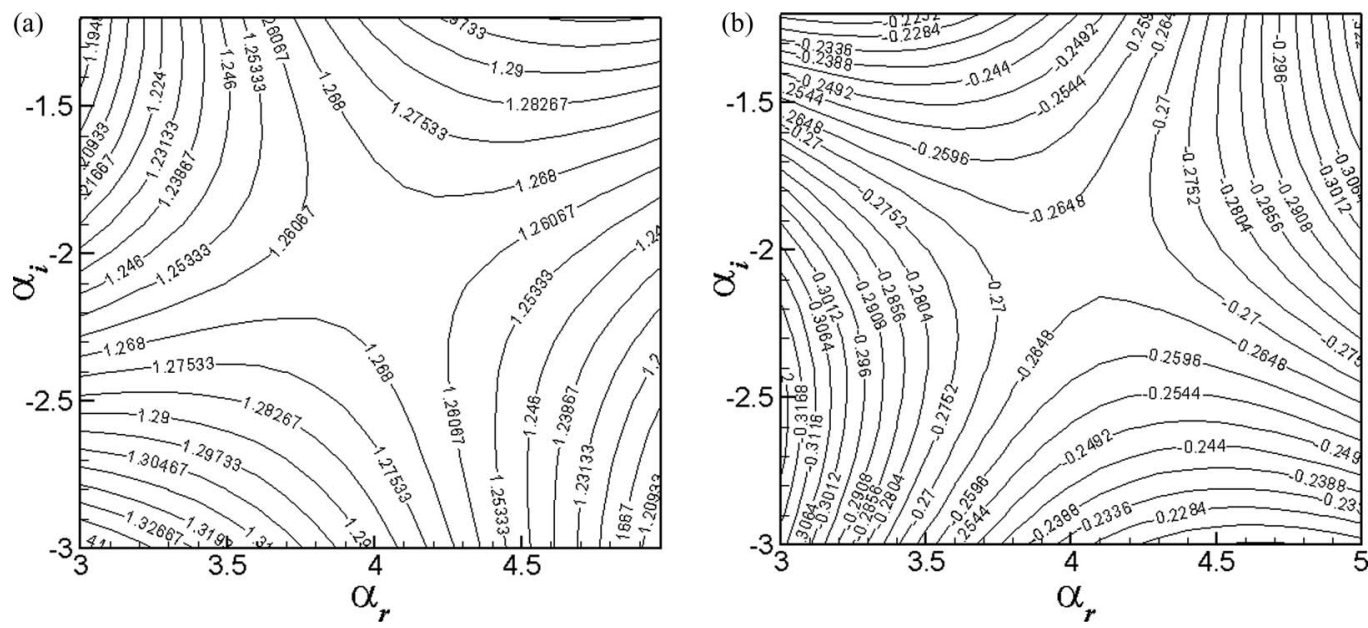

FIG. 5. Isocontours of (a) $\omega_{r}$ and (b) $\omega_{i}$ in the complex wavenumber plane for a single component system equivalent to the double-diffusive system of Figure 4 . The parameters are $\mathrm{Re}=200, \mathrm{Sc}=9.091, h=0.6, q=0.1, R_{S}=3.5$, and $R_{f}=0$. At the saddle point, $\omega_{0}$ is $1.265-0.266 \mathbf{i}$, i.e., the flow is not absolutely unstable. In fact, the zero group velocity mode is damped out rapidly. 

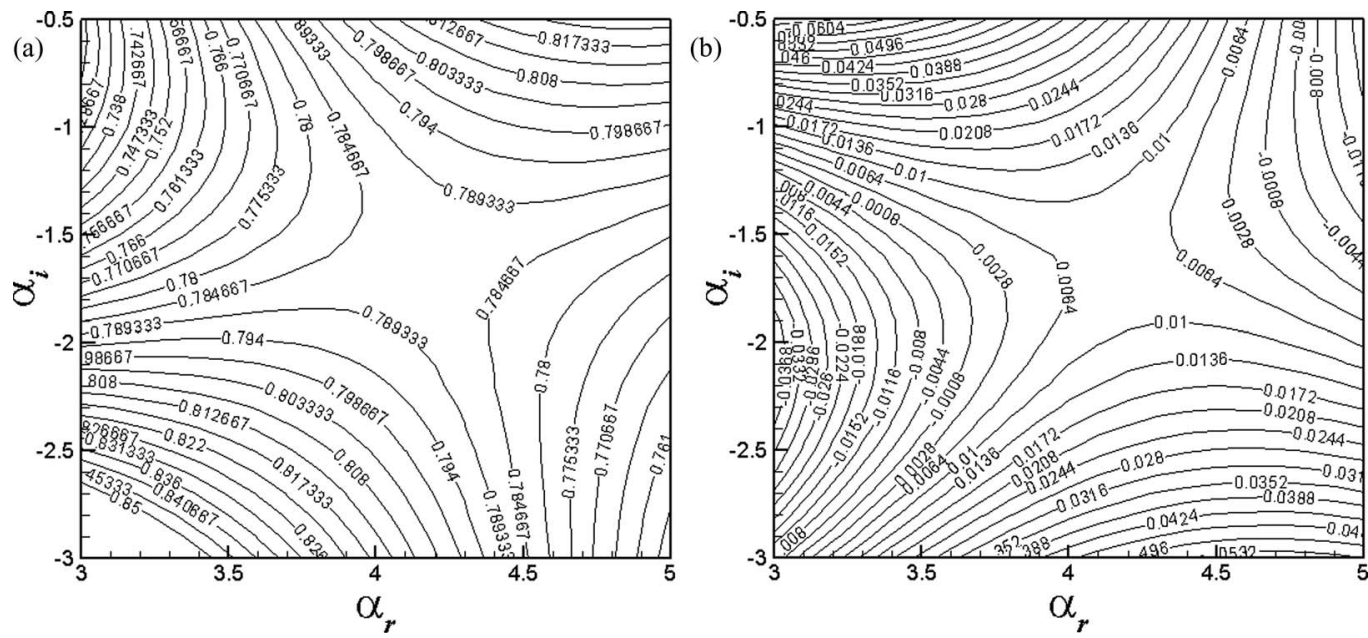

FIG. 6. Isocontours of (a) $\omega_{r}$ and (b) $\omega_{i}$ in the complex wavenumber plane. The parameters are $\mathrm{Re}=200, \mathrm{Sc}=50, \delta=10$, $h=0.6, q=0.1, R_{s}=4$, and $R_{f}=0$. At the saddle point, $\omega_{0}$ is $0.787+0.007 \mathbf{i}$.
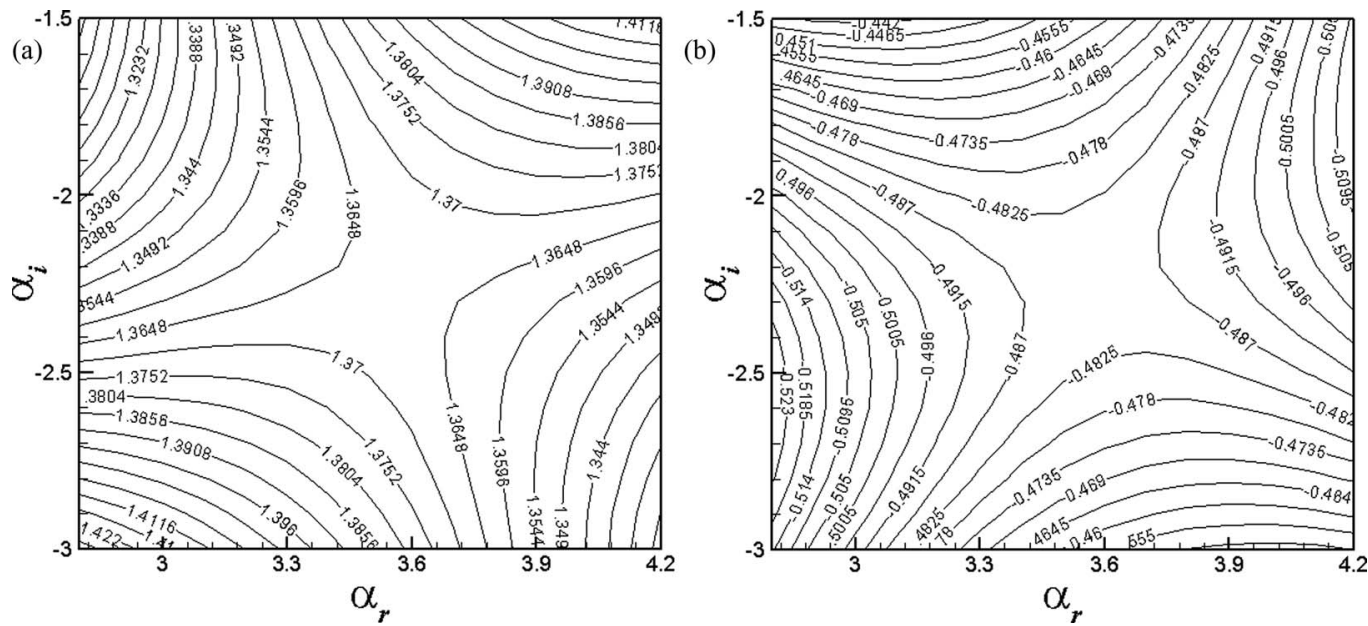

FIG. 7. Isocontours of (a) $\omega_{r}$ and (b) $\omega_{i}$ in the complex wavenumber plane. The parameters are the same as those used to generate the $C_{\mathrm{DD}}$ curve of Figure 3 . The absolute frequency $\omega_{0}$, i.e., $\omega$ at the saddle point, is $1.366-0.485 \mathbf{i}$, showing that the flow is not absolutely unstable.
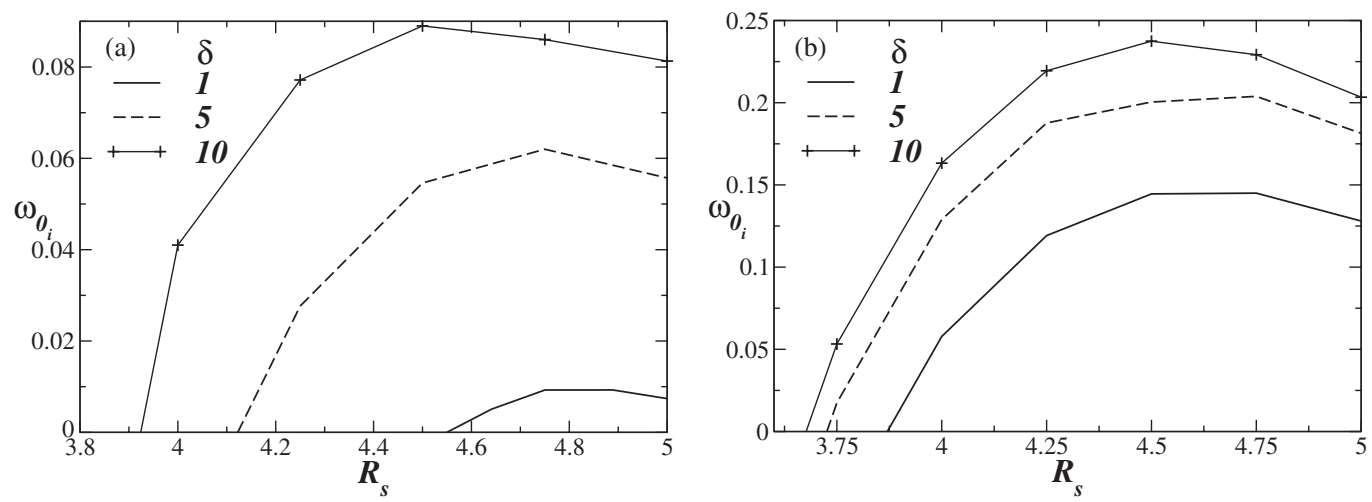

FIG. 8. Variation of the absolute growth rate with $R_{s}$ for different $\delta$ values for (a) $q=0.1$ and (b) $q=0.05$, respectively. The rest of the parameter values are $R_{f}=-1, \operatorname{Re}=200, \mathrm{Sc}=50$, and $h=0.6$. The absolute instability is stronger for a DD case than for an SC one. 

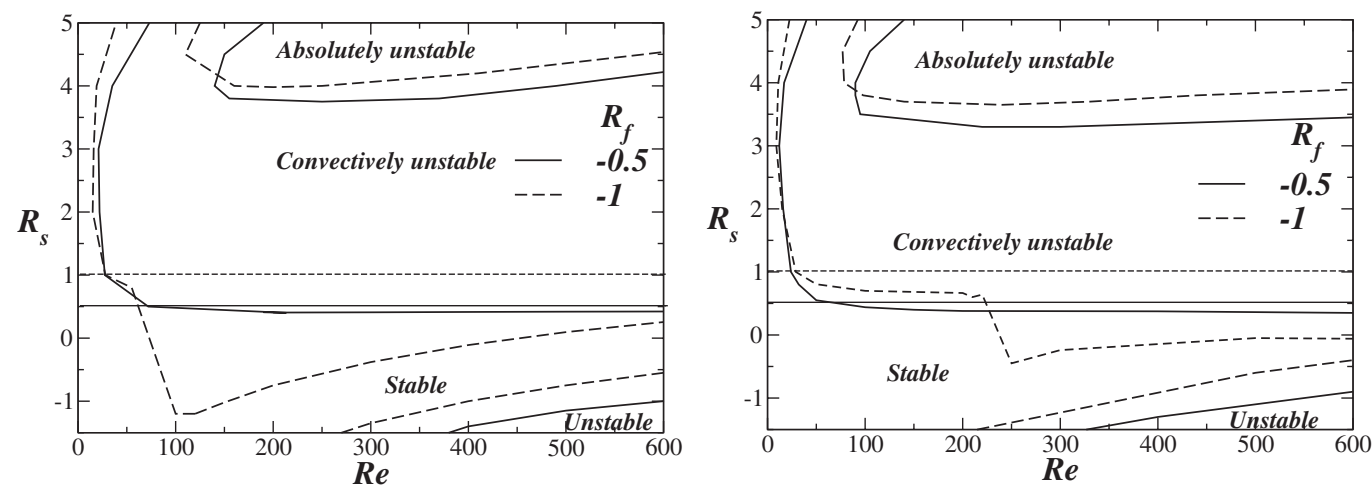

FIG. 9. Stability diagrams showing the regions of convective and absolute instabilities in $R_{s}-R e$ space for (a) $q=0.1$ and (b) $q=0.05$, respectively. The rest of the parameter values are $\mathrm{Sc}=50, \delta=10, h=0.6$, and $q=0.1$. The horizontal lines in each case show the location where $R_{s}+R_{f}=0$. Above this line, the average viscosity increases as we move from the centerline of the channel towards the wall.

seen above a Reynolds number of above 100, when the viscosity stratification is high, i.e., $R_{s}+R_{f}$ larger than about 3 for this choice of parameters.

\section{SUMMARY}

The double-diffusive spatio-temporal instability of the pressure-driven three-layer flow of two miscible, Newtonian and incompressible fluids of equal density and different viscosities through a channel is studied. The two fluids consist of different concentrations of two scalar species of different diffusion rates. The viscosity is modelled as an exponential function of both scalars, and a Briggstype method is used in order to investigate the convective and absolute nature of the instabilities. The linear stability equations for complex wavenumber and frequency are solved using Chebyshev spectral collocation method on a stretched grid, which provides a larger number of grid points in the mixed layers and in the near wall regions.

The main finding is a large enhancement in both the parameter range and strength of absolute instability, when one uses a double-diffusive rather than a single component system. This happens when the slower diffusing species is destabilizing, i.e., contributes a viscosity which is higher near the channel walls than in the centerline. In the more usually studied gravity-driven DD systems, when the slower diffusing species is destabilizing, and the faster one is stabilizing, concentration perturbations in the latter diffuse away sooner than those in the former, leaving a net driving of the instability by the slower diffusing species. This simple argument is not sufficient to explain the present findings, since the DD system shows a far higher absolute growth rate than even a SC system made up of only the slower diffusing species. Furthermore, increasing the contrast in diffusion rates makes the flow more convectively unstable, even while we decrease the net stratification of the system, and therefore expect a net stabilization.

Since absolute instabilities are typically catastrophic, one may extrapolate that good mixing and an early transition to turbulence would be natural consequences. In industrial double-diffusive flows, a Reynolds number of the order of a thousand is realistic. Since absolute instabilities are likely to dominate over convective ones, in such flows, if mixing is desired, one might thus benefit from imposing such a viscosity stratification. We hope the present predictions will motivate experimental verification of viscosity-driven DD channel flows.

\section{ACKNOWLEDGMENTS}

K.S. gratefully acknowledges the support from the Indian Institute of Technology Hyderabad, India. 
${ }^{1}$ D. D. Joseph, R. Bai, K. P. Chen, and Y. Y. Renardy, "Core-annular flows,” Ann. Rev. Fluid Mech. 29, 65 (1997).

${ }^{2}$ P. A. M. Boomkamp and R. H. M. Miesen, "Classification of instabilities in parallel two-phase flow," Int. J. Multiphase Flow 22, 67 (1996).

${ }^{3}$ M. J. South and A. P. Hooper, "Linear growth in two-fluid plane Poiseuille flow," J. Fluid Mech. 381, 121 (1999).

${ }^{4}$ R. Govindarajan, "Effect of miscibility on the linear instability of two-fluid channel flow," Int. J. Multiphase Flow 30, 1177 (2004).

${ }^{5}$ B. Selvam, S. Merk, R. Govindarajan, and E. Meiburg, "Stability of miscible core-annular flows with viscosity stratification,” J. Fluid Mech. 592, 23 (2007).

${ }^{6}$ S. V. Malik and A. P. Hooper, "Linear stability and energy growth of viscosity stratified flows," Phys. Fluids 17, 024101 (2005).

${ }^{7}$ M. d'Olce, J. Martin, N. Rakotomalala, D. Salin, and L. Talon, "Pearl and mushroom instability patterns in two miscible fluids' core annular flows," Phys. Fluids 20, 024104 (2008).

${ }^{8}$ K. C. Sahu, H. Ding, P. Valluri, and O. K. Matar, "Linear stability analysis and numerical simulation of miscible channel flows," Phys. Fluids 21, 042104 (2009).

${ }^{9}$ B. Selvam, L. Talon, L. Lesshafft, and E. Meiburg, "Convective/absolute instability in miscible core-annular flow. Part 2. Numerical simulations and nonlinear global modes," J. Fluid Mech. 618, 323 (2009).

${ }^{10}$ P. Huerre and P. A. Monkewitz, "Local and global instabilities in spatially developing flows," Ann. Rev. Fluid Mech. 22, 473 (1990).

${ }^{11}$ J.-M. Chomaz, "Global instabilities in spatially developing flows: Non-normality and nonlinearity," Ann. Rev. Fluid Mech. 37, 357 (2005).

${ }^{12}$ P. Huerre and M. Rossi, in Hydrodynamics and Nonlinear Instabilities, edited by C. Godreche and P. Manneville (Cambridge University Press, Cambridge, England, 1998), pp. 81-288.

${ }^{13}$ J. S. Turner, "Double-diffusive phenomena," Ann. Rev. Fluid Mech. 6, 37 (1974).

${ }^{14}$ H. E. Huppert, "On the stability of a series of double-diffusive layers," Deep-Sea Res. Oceanogr. Abstr. 18(10), 1005 (1971).

${ }^{15}$ B. D. May and D. E. Kelley, "Effect of baroclinicity on double-diffusive interleaving," J. Phys. Oceanogr. 27, 1997 (1997).

${ }^{16}$ K. C. Sahu and R. Govindarajan, "Linear stability of double-diffusive two-fluid channel flow," J. Fluid Mech. 687, 529 (2011).

${ }^{17}$ R. J. Briggs, "Electron stream interaction with plasmas," Research Monograph No. 29 (MIT, Cambridge, 1964).

${ }^{18}$ P. J. Schmid and D. S. Henningson, Stability and Transition in Shear Flows (Springer, New York, 2001).

${ }^{19}$ C. Canuto, M. Y. Hussaini, A. Quarteroni, and T. Zang, Spectral Methods in Fluid Dynamics, 1st ed. (Springer-Verlag, Amsterdam, 1987), pp. 65-70.

${ }^{20}$ A. Bers, in Handbook of Plasma Physics, edited by M. N. Rosenbluth and R. Z. Sagdeev (North-Holland, Amsterdam, 1983), Vol. 1, pp. 451-517.

${ }^{21}$ I. Vihinen, A. M. Honohan, and S. P. Lin, "Image of absolute instability in a liquid jet," Phys. Fluids 9(11), 3117 (1997).

${ }^{22}$ W. O. Criminale, T. L. Jackson, and R. D. Joslin, Theory and Computation of Hydrodynamic Stability (Cambridge University Press, Cambridge, England, 2003).

${ }^{23}$ L. Kaiktsis and P. A. Monkewitz, "Global destabilization of flow over a backward-facing step," Phys. Fluids 15(12), 3647 (2003). 